\title{
Some results on fixed point theory for a class of generalized nonexpansive mappings
}

\author{
Bijoy Patir ${ }^{1}$, Nilakshi Goswami ${ }^{1}$ and Vishnu Narayan Mishra ${ }^{2 *}$
}

\author{
"Correspondence: \\ vishnunarayanmishra@gmail.com \\ ${ }^{2}$ Department of Mathematics, Indira \\ Gandhi National Tribal University, \\ Amarkantak, India \\ Full list of author information is \\ available at the end of the article
}

\begin{abstract}
In this paper, we introduce a new class of generalized nonexpansive mappings which is wider than the class of mappings satisfying (C) condition. Different properties and some fixed point results for these mappings are obtained here. The convergence of some iteration schemes to the fixed point is also discussed with suitable examples.
\end{abstract}

MSC: $47 \mathrm{H} 10 ; 54 \mathrm{H} 25$

Keywords: Nonexpansive mapping; (C) condition; Fixed point

\section{Introduction and preliminaries}

The generalization of nonexpansive mappings and the study of related fixed point theorems with different practical applications in nonlinear functional analysis have found great importance during the recent decades $[1-6,8-10,12,14,15,19,22-24,26,27,32]$. Several prominent authors $[11,13,16,20,21,25,28,29,31]$ have contributed immensely in this field, and different new classes of mappings with interesting properties have been developed in this context.

In 2008, Suzuki defined a class of generalized nonexpansive mappings on a nonempty subset $C$ of a Banach space $X$. Such type of mappings was called the class of mappings satisfying the condition $(C)$ (see $[17,18,30])$. For a nonempty bounded and convex subset $C$, every self-mapping $T$ on $C$ satisfying $(C)$ condition has an almost fixed point sequence [15]. In 2011, Falset et al. [15] introduced two new classes of generalized nonexpansive mappings which are wider than those satisfying $(\mathrm{C})$ condition, but preserving their fixed point properties.

In this paper, we introduce a new class of mappings which is larger than the class satisfying the condition (C). We study the existence of fixed points for this type of mappings with some examples.

First we present some basic concepts.

Definition 1.1 (see [30]) Let $C$ be a nonempty subset of a Banach space $X$. A mapping $T: C \longrightarrow X$ is said to be nonexpansive if $\|T x-T y\| \leq\|x-y\|$ for all $x, y \in C$.

Definition 1.2 (see [30]) For a nonempty subset $C$ of a Banach space $X$, a mapping $T$ : $C \longrightarrow X$ is called quasi nonexpansive if $\|T x-z\| \leq\|x-z\|$ for all $x \in C$ and $z \in F(T)$ (where $F(T)$ denotes the set of all fixed points of $T$ ).

(c) The Author(s) 2018. This article is distributed under the terms of the Creative Commons Attribution 4.0 International License (http://creativecommons.org/licenses/by/4.0/), which permits unrestricted use, distribution, and reproduction in any medium, provided you give appropriate credit to the original author(s) and the source, provide a link to the Creative Commons license, and indicate if changes were made. 
Definition 1.3 (see [30]) For a nonempty subset $C$ of a Banach space $X$, a mapping $T$ : $C \rightarrow X$ is said to satisfy the condition (C) on $C$ if $\frac{1}{2}\|x-T x\| \leq\|x-y\|$ implies $\|T x-T y\| \leq$ $\|x-y\|$ for all $x, y \in C$.

Clearly, every nonexpansive mapping satisfies the condition (C) on $C$. But there are also some noncontinuous mappings satisfying the condition (C) (see [15]).

Definition 1.4 (see [15]) For a nonempty subset $C$ of a Banach space $X$ and $\lambda \in(0,1)$, a mapping $T: C \longrightarrow X$ is said to satisfy $\left(C_{\lambda}\right)$-condition on $C$ if $\lambda\|x-T x\| \leq\|x-y\|$ implies $\|T x-T y\| \leq\|x-y\|$ for all $x, y \in C$.

Definition 1.5 (see [15]) If $C$ is a closed convex and bounded subset of $X$, and a selfmapping $T$ on $C$ is nonexpansive, then there exists a sequence $x_{n}$ in $C$ such that $\| x_{n}-$ $T x_{n} \| \longrightarrow 0$. Such a sequence is called almost fixed point sequence for $T$.

Definition 1.6 (see [15]) Let $C$ be a nonempty subset of a Banach space $X$ and $\left\{x_{n}\right\}$ be a bounded sequence in $X$. For each $x \in X$,

(i) asymptotic radius of $\left\{x_{n}\right\}$ at $x$ is defined by $r\left(x,\left\{x_{n}\right\}\right)=\limsup _{n \rightarrow \infty}\left\|x_{n}-x\right\|$.

(ii) asymptotic radius of $\left\{x_{n}\right\}$ relative to $C$ is defined by $r\left(C,\left\{x_{n}\right\}\right)=\inf r\left(x,\left\{x_{n}\right\}\right): x \in C$.

(iii) asymptotic center of $\left\{x_{n}\right\}$ relative to $C$ is defined by $A\left(C,\left\{x_{n}\right\}\right)=\left\{x \in C: r\left(x,\left\{x_{n}\right\}\right)=r\left(C,\left\{x_{n}\right\}\right)\right\}$. We note that $A\left(C,\left\{x_{n}\right\}\right)$ is nonempty. Again, if $X$ is uniformly convex, then $A\left(C,\left\{x_{n}\right\}\right)$ has exactly one point.

Definition 1.7 (see [30]) A Banach space $X$ is said to satisfy the Opial property if, for every sequence $\left\{x_{n}\right\}$ in $X$ with $x_{n} \longrightarrow z$ (weakly), we have

$$
\lim _{n \rightarrow \infty} \inf \left\|x_{n}-z\right\|<\lim _{n \rightarrow \infty} \inf \left\|x_{n}-y\right\|
$$

whenever $y \neq z$.

For example, $l^{p}$ spaces $(1<p<\infty)$ satisfy this condition.

\section{Methods}

We apply both analytic as well as fixed point theoretical method to prove our results. Different existing methods in the literature (refer to $[15,20,30]$ ) as well as some new approaches are also taken.

\section{Results and discussion}

We construct the following class of mappings.

Definition 3.1 Let $C$ be a nonempty subset of a Banach space $X$. Let $\gamma \in[0,1]$ and $\mu \in$ $\left[0, \frac{1}{2}\right]$ such that $2 \mu \leq \gamma$. A mapping $T: C \longrightarrow X$ is said to satisfy the condition $B_{\gamma, \mu}$ on $C$ if, for all $x, y$ in $C$,

$$
\gamma\|x-T x\| \leq\|x-y\|+\mu\|y-T y\|
$$

implies $\|T x-T y\| \leq(1-\gamma)\|x-y\|+\mu(\|x-T y\|+\|y-T x\|)$.

Clearly, this class includes the class of nonexpansive mappings (for $\gamma=\mu=0$ ). 
Also, if a mapping satisfies the condition (C), then it will satisfy the condition $B_{\gamma, \mu}$ for $\gamma=\mu=0$.

$$
\begin{aligned}
& \text { As } \frac{1}{2}\|x-T x\| \leq\|x-y\| \Rightarrow\|T x-T y\| \leq\|x-y\| \text { for (C) condition, } \\
& \text { so, clearly, }\|T x-T y\| \leq(1-\gamma)\|x-y\|+\mu(\|x-T y\|+\|y-T x\|) \text { for } \gamma=\mu=0 .
\end{aligned}
$$

But the converse is not true.

Example 3.2 Let $T:[0,2] \longrightarrow \mathbb{R}$ be defined by

$$
T(x)= \begin{cases}0 & \text { if } x \neq 2 \\ 1 & \text { if } x=2\end{cases}
$$

Now, for $x=1.2, y=2, \frac{1}{2}\|T x-x\|=0.6<0.8=\|x-y\|$. But $\|T x-T y\|=1 \nless 0.8=\|x-y\|$. So, the condition $(C)$ is not satisfied.

For $x \neq 2, y \neq 2$, obviously $T$ satisfies $B_{\gamma, \mu}$ condition for $\gamma=1$ and $\mu=\frac{1}{2}$.

Again, let $x \neq 2, y=2$.

Then $\|T x-T y\|=1$ and

$$
\begin{aligned}
& (1-\gamma)\|x-y\|+\mu(\|x-T y\|+\|y-T x\| \\
& \quad=\frac{1}{2}\|x-1\|+1 \quad\left(\text { for } \gamma=1, \mu=\frac{1}{2}\right) \\
& >1=\|T x-T y\| .
\end{aligned}
$$

For $x=2, y \neq 2$,

$$
\begin{aligned}
& \|T x-T y\|=1<(1-\gamma)\|x-y\|+\mu(\|x-T y\|+\|y-T x\|) \\
& \left(\text { for } \gamma=1, \mu=\frac{1}{2}\right) \text { as above. }
\end{aligned}
$$

For $x=2, y=2, B_{\gamma, \mu}$ condition (for $\gamma=1, \mu=\frac{1}{2}$ ) is obviously satisfied by $T$.

The following lemma shows that $T$ satisfying $B_{\gamma, \mu}$ condition is also quasi-nonexpansive.

Lemma 3.3 For a nonempty subset $C$ of a Banach space $X$, let $T: C \longrightarrow X$ be a mapping satisfying $B_{\gamma, \mu}$ condition. If $z$ is a fixed point of $T$ on $C$, then for all $x \in C$,

$$
\|z-T x\| \leq\|z-x\|
$$

Proof We have

$$
\gamma\|z-T z\|=0 \leq\|z-x\|+\mu\|x-T x\| .
$$

By $B_{\gamma, \mu}$ condition,

$$
\|T z-T x\| \leq(1-\gamma)\|z-x\|+\mu(\|x-T z\|+\|z-T x\|)
$$




$$
\begin{gathered}
=(1-\gamma)\|z-x\|+\mu(\|x-z\|+\|z-T x\|) \\
\Rightarrow \quad\|z-T x\| \leq\left(\frac{1-\gamma+\mu}{1-\mu}\right)\|z-x\| \leq\|z-x\| \quad(\text { as } 2 \mu \leq \gamma)
\end{gathered}
$$

showing that $T$ is quasi-nonexpansive.

However, the converse of Lemma 3.3 does not hold in general.

Example 3.4 Let $T$ be a mapping on $[0,4]$ defined by

$$
T(x)= \begin{cases}0 & \text { if } x \neq 4 \\ 3 & \text { if } x=4\end{cases}
$$

Clearly $T$ has a fixed point at $x=0$, and also $\|T(x)\| \leq\|x\| \forall x \in[0,4]$.

Hence, $T$ is quasi-nonexpansive.

We show that $T$ does not satisfy the condition $B_{\gamma, \mu}$.

For $x=4, y=3$,

$$
\gamma\|x-T x\|=\gamma \leq 1+3 \mu=\|4-3\|+\mu\|3-T(3)\| .
$$

But

$$
\|T x-T y\|=\|T(4)-T(3)\|=3
$$

and

$$
\begin{aligned}
& (1-\gamma)\|x-y\|+\mu(\|x-T y\|+\|y-T x\|) \\
& \quad=1-\gamma+4 \mu \\
& \quad \leq 1-\gamma+2 \gamma \quad(2 \mu \leq \gamma) \\
& \quad<3 \quad(\text { as } \gamma \in[0,1]) \\
& =\|T x-T y\| .
\end{aligned}
$$

So, $B_{\gamma, \mu}$ condition is not satisfied.

The following are some basic properties of mappings which satisfy the condition $B_{\gamma, \mu}$ on $C$.

Proposition 3.5 Let $C$ be a nonempty subset of a Banach space X. Let $T: C \longrightarrow C$ satisfy the condition $B_{\gamma, \mu}$ on $C$. Then, for all $x, y \in C$ and for $c \in[0,1]$,

(i) $\left\|T x-T^{2} x\right\| \leq\|x-T x\|$,

(ii) at least one of the following ((a) and (b)) holds:

(a) $\frac{c}{2}\|x-T x\| \leq\|x-y\|$

(b) $\frac{c}{2}\left\|T x-T^{2} x\right\| \leq\|T x-y\|$.

The condition (a) implies $\|T x-T y\| \leq\left(1-\frac{c}{2}\right)\|x-y\|+\mu(\|x-T y\|+\|y-T x\|)$ and the condition (b) implies $\left\|T^{2} x-T y\right\| \leq\left(1-\frac{c}{2}\right)\|T x-y\|+\mu\left(\|T x-T y\|+\left\|y-T^{2} x\right\|\right)$. 
(iii) $\|x-T y\| \leq(3-c)\|x-T x\|+\left(1-\frac{c}{2}\right)\|x-y\|+\mu(2\|x-T x\|+\|x-T y\|+\|y-T x\|+$ $\left.2\left\|T x-T^{2} x\right\|\right)$.

Proof (i) We have, for all $x \in C$,

$$
\gamma\|x-T x\| \leq\|x-T x\|+\mu\left\|T x-T^{2} x\right\| .
$$

So, by the condition $B_{\gamma, \mu}$ (replacing $y$ by $T x$ ),

$$
\begin{aligned}
&\left\|T x-T^{2} x\right\| \leq(1-\gamma)\|x-T x\|+\mu\left\|x-T^{2} x\right\| \\
& \leq(1-\gamma)\|x-T x\|+\mu\|x-T x\|+\mu\left\|T x-T^{2} x\right\| \\
& \Rightarrow \quad\left\|T x-T^{2} x\right\| \leq \frac{1-\gamma+\mu}{1-\mu}\|x-T x\| \leq\|x-T x\| .
\end{aligned}
$$

(ii) We assume on the contrary that $\frac{c}{2}\|x-T x\|>\|x-y\|$ and $\frac{c}{2}\left\|T x-T^{2} x\right\|>\|T x-y\|$ for some $x, y \in C$.

Now,

$$
\begin{aligned}
\|x-T x\| & \leq\|x-y\|+\|y-T x\| \\
& <\frac{c}{2}\|x-T x\|+\frac{c}{2}\left\|T x-T^{2} x\right\| \\
& \leq \frac{c}{2}\|x-T x\|+\frac{c}{2}\|x-T x\| \quad(\text { by }(i)) \\
& \leq\|x-T x\| \quad(\text { since } c \leq 1),
\end{aligned}
$$

i.e.,

$$
\|x-T x\|<\|x-T x\|
$$

which is impossible.

So, at least one of (a) and (b) holds.

(iii) $\|x-T y\| \leq\|x-T x\|+\|T x-T y\|$

If (ii)(a) holds,

$$
\begin{aligned}
\|x-T y\| \leq & \|x-T x\|+\left(1-\frac{c}{2}\right)\|x-y\|+\mu(\|x-T y\|+\|y-T x\|) \\
\leq & (3-c)\|x-T x\|+\left(1-\frac{c}{2}\right)\|x-y\| \\
& +\mu\left(2\|x-T x\|+\|x-T y\|+\|y-T x\|+2\left\|T x-T^{2} x\right\|\right) .
\end{aligned}
$$

If (ii)(b) holds,

$$
\begin{aligned}
\|x-T y\| & \leq\|x-T x\|+\left\|T x-T^{2} x\right\|+\left\|T^{2} x-T y\right\| \\
& \leq\|x-T x\|+\left(1-\frac{c}{2}\right)\|T x-x\|+\mu\left(\|T x-T x\|+\left\|x-T^{2} x\right\|\right)
\end{aligned}
$$




$$
\begin{aligned}
& +\left(1-\frac{c}{2}\right)\|T x-y\|+\mu\left(\|T x-T y\|+\left\|y-T^{2} x\right\|\right) \\
= & (3-c)\|x-T x\|+\left(1-\frac{c}{2}\right)\|x-y\| \\
& +\mu\left(\left\|x-T^{2} x\right\|+\|T x-T y\|+\left\|y-T^{2} x\right\|\right) \\
\leq & (3-c)\|x-T x\|+\left(1-\frac{c}{2}\right)\|x-y\|+\mu\left(\|x-T x\|+\left\|T x-T^{2} x\right\|\right. \\
& \left.+\|x-T x\|+\|x-T y\|+\|y-T x\|+\left\|T x-T^{2} x\right\|\right) \\
= & (3-c)\|x-T x\|+\left(1-\frac{c}{2}\right)\|x-y\| \\
& +\mu\left(2\|x-T x\|+\|x-T y\|+\|y-T x\|+2\left\|T x-T^{2} x\right\|\right) .
\end{aligned}
$$

Proposition 3.6 Let $C$ be a nonempty convex and bounded subset of a Banach space $X$ and $T$ be a self-mapping on $C$. We assume that $T$ satisfies the condition $B_{\gamma, \mu}$ on $C$. For $x_{0} \in C$, let a sequence $\left\{x_{n}\right\}$ in $C$ be defined by

$$
x_{n+1}=\lambda T x_{n}+(1-\lambda) x_{n}
$$

where $\lambda \in[\gamma, 1)-\{0\}, n \in \mathbb{N} \cup\{0\}$. Then $\left\|T x_{n}-x_{n}\right\| \longrightarrow 0$ as $n \longrightarrow \infty$.

Proof Since $\lambda \geq \gamma$, we have

$$
\begin{aligned}
\gamma\left\|x_{n}-T x_{n}\right\| & \leq \lambda\left\|x_{n}-T x_{n}\right\| \\
& =\left\|x_{n}-x_{n+1}\right\| \quad(\text { by }(3.1))
\end{aligned}
$$

i.e.,

$$
\gamma\left\|x_{n}-T x_{n}\right\| \leq\left\|x_{n}-x_{n+1}\right\|+\mu\left\|x_{n+1}-T x_{n+1}\right\|
$$

So, by the condition $B_{\gamma, \mu}\left(\right.$ for $\left.y=x_{n+1}\right)$,

$$
\begin{aligned}
& \left\|T x_{n}-T x_{n+1}\right\| \leq(1-\gamma)\left\|x_{n}-x_{n+1}\right\|+\mu\left(\left\|x_{n}-T x_{n+1}\right\|+\left\|x_{n+1}-T x_{n}\right\|\right) \\
& \Rightarrow \quad\left\|T x_{n}-\frac{1}{\lambda}\left(x_{n+2}-(1-\lambda) x_{n+1}\right)\right\| \\
& \quad \leq\left\|x_{n}-x_{n+1}\right\|+\mu\left(\left\|x_{n}-\frac{1}{\lambda}\left(x_{n+2}-(1-\lambda) x_{n+1}\right)\right\|+\left\|x_{n+1}-T x_{n}\right\|\right),
\end{aligned}
$$

from which we get

$$
\begin{aligned}
& (1-\mu) \lim _{n \rightarrow \infty}\left\|T x_{n}-x_{n}\right\| \leq 0 \\
& \Rightarrow \quad \lim _{n \rightarrow \infty}\left\|T x_{n}-x_{n}\right\|=0 \quad(\text { since } \mu \neq 1) .
\end{aligned}
$$

Corollary 3.7 Let C be a closed convex and bounded subset of a Banach space X. Let T be a self-mapping on $C$ satisfying the condition $B_{\gamma, \mu}$. Then the sequence $\left\{x_{n}\right\}$ as defined above is an almost fixed point sequence. 
For a nonempty compact convex subset $C$ of $X$, we have the following fixed point result.

Theorem 3.8 Let $C$ be a compact and convex subset of a Banach space X. Let $T$ be a self-mapping on $C$ satisfying the condition $B_{\gamma, \mu}$. For $x_{0} \in C$, let $\left\{x_{n}\right\}$ be a sequence in $C$ as defined in Proposition 3.6, where $\gamma$ is sufficiently small. Then $\left\{x_{n}\right\}$ converges strongly to a fixed point of $T$.

Proof Since $C$ is compact, there exists a subsequence $\left\{x_{n_{j}}\right\}$ of $\left\{x_{n}\right\}$ and $z \in C$ such that $\left\{x_{n_{j}}\right\}$ converges to $z$ (see [31]).

Now, by Proposition 3.5(ii), for $\gamma=\frac{c}{2}, c \in[0,1]$

$$
\begin{aligned}
& \gamma\left\|x_{n_{j}}-T x_{n_{j}}\right\| \leq\left\|x_{n_{j}}-z\right\| \\
& \Rightarrow \quad \gamma\left\|x_{n_{j}}-T x_{n_{j}}\right\| \leq\left\|x_{n_{j}}-z\right\|+\mu\|z-T z\| .
\end{aligned}
$$

So, by the condition $B_{\gamma, \mu}$,

$$
\left\|T x_{n_{j}}-T z\right\| \leq(1-\gamma)\left\|x_{n_{j}}-z\right\|+\mu\left(\left\|x_{n_{j}}-T z\right\|+\left\|z-T x_{n_{j}}\right\|\right) .
$$

Again,

$$
\begin{aligned}
& \left\|x_{n_{j}}-T z\right\| \\
& \quad \leq\left\|x_{n_{j}}-T x_{n_{j}}\right\|+\left\|T x_{n_{j}}-T z\right\| \\
& \quad \leq\left\|x_{n_{j}}-T x_{n_{j}}\right\|+(1-\gamma)\left\|x_{n_{j}}-z\right\|+\mu\left(\left\|x_{n_{j}}-T z\right\|+\left\|z-T x_{n_{j}}\right\|\right) \quad \text { (by (3.2)) } \\
& \quad \leq\left\|x_{n_{j}}-T x_{n_{j}}\right\|+(1-\gamma)\left\|x_{n_{j}}-z\right\|+\mu\left(\left\|x_{n_{j}}-T z\right\|+\left\|z-x_{n_{j}}\right\|+\left\|x_{n_{j}}-T x_{n_{j}}\right\|\right) .
\end{aligned}
$$

So, taking $n_{j} \longrightarrow \infty$ and using Proposition 3.6, we get

$$
\begin{aligned}
& (1-\mu)\|z-T z\| \leq 0 \\
& \quad \Rightarrow \quad T z=z, \quad(\text { since } \mu \neq 1)
\end{aligned}
$$

showing that $z$ is a fixed point for $T$. Now,

$$
\begin{aligned}
\left\|x_{n+1}-z\right\| & \leq \lambda\left\|T x_{n}-z\right\|+(1-\lambda)\left\|x_{n}-z\right\| \\
& \leq \lambda\left\|x_{n}-z\right\|+(1-\lambda)\left\|x_{n}-z\right\| \quad(\text { by Lemma 3.3) } \\
& =\left\|x_{n}-z\right\| \quad \text { for all } n \in \mathbb{N} \cup\{0\}
\end{aligned}
$$

Thus, $\left\{\left\|x_{n}-z\right\|\right\}$ is a monotonically decreasing sequence of nonnegative real numbers and will converge to some real, say $u$. Now,

$$
\begin{gathered}
\left\|x_{n}-T z\right\| \leq\left\|x_{n}-T x_{n}\right\|+(1-\gamma)\left\|x_{n}-z\right\|+\mu\left(\left\|x_{n}-T z\right\|+\left\|z-x_{n}\right\|+\left\|x_{n}-T x_{n}\right\|\right) \\
\Rightarrow \quad\left\|x_{n}-z\right\| \leq\left\|x_{n}-T x_{n}\right\|+(1-\gamma)\left\|x_{n}-z\right\|+\mu\left(2\left\|x_{n}-z\right\|+\left\|x_{n}-T x_{n}\right\|\right) .
\end{gathered}
$$


Taking limit as $n \longrightarrow \infty$, we get $u \leq(1-\gamma) u+\mu(2 u) \Rightarrow(\gamma-2 \mu) u \leq 0$, which is possible only for $u=0$, since $2 \mu \leq \gamma$. Hence, $\left\{x_{n}\right\}$ converges strongly to $z$.

Example 3.9 On the subset $C=[0,4]$ of the Banach space $\mathbb{R}$, define $T$ by

$$
T(x)= \begin{cases}0 & \text { if } x \neq 4 \\ 2 & \text { if } x=4\end{cases}
$$

Then $T$ satisfies the condition $B_{\gamma, \mu}$. Let $\left\{x_{n}\right\}$ be a sequence in $C$ defined as in Proposition 3.6.

Suppose, $x_{0}=3$. Then we have $x_{n}=3(1-\lambda)^{n}$, converging to 0 as $n \longrightarrow \infty$. Clearly, 0 is the fixed point of $T$.

Theorem 3.10 Let $C$ be a weakly compact and convex subset of a uniformly convex Banach space $X$. Let $T$ be a self-mapping on $C$ satisfying the condition $B_{\gamma, \mu}$. Then $T$ has a fixed point.

Proof Consider the sequence $\left\{x_{n}\right\}$ in $C$ as defined in Proposition 3.6. Then lim $\sup _{n} \| T x_{n}-$ $x_{n} \|=0$. As in [15], let $g$ be a continuous convex function from $C$ into $[0, \infty)$ defined by

$$
g(x)=\lim _{n \rightarrow \infty} \sup \left\|x_{n}-x\right\|
$$

for all $x \in C$.

Again, since $C$ is weakly compact and $g$ is weakly lower semi-continuous, there is $z \in C$ such that

$$
g(z)=\min \{g(x): x \in C\}
$$

Now, by Proposition 3.5(iii) (for $\gamma=\frac{c}{2}$ ),

$$
\begin{aligned}
\left\|x_{n}-T z\right\| \leq & (3-2 \gamma)\left\|x_{n}-T x_{n}\right\|+(1-\gamma)\left\|x_{n}-z\right\|+\mu\left(2\left\|x_{n}-T x_{n}\right\|\right. \\
& \left.+\left\|x_{n}-T z\right\|+\left\|x_{n}-z\right\|+\left\|x_{n}-T x_{n}\right\|+2\left\|x_{n}-T x_{n}\right\|\right) .
\end{aligned}
$$

So,

$$
\begin{aligned}
& (1-\mu) \lim _{n \rightarrow \infty} \sup \left\|x_{n}-T z\right\| \leq(1-\gamma+\mu) \lim _{n \rightarrow \infty} \sup \left\|x_{n}-z\right\| \\
& \Rightarrow \lim _{n \rightarrow \infty} \sup \left\|x_{n}-T z\right\| \leq \frac{(1-\gamma+\mu)}{(1-\mu)} \lim _{n \rightarrow \infty} \sup \left\|x_{n}-z\right\| \\
& \Rightarrow g(T z) \leq g(z) .
\end{aligned}
$$

Since $g(z)$ is the minimum, $g(T z)=g(z)$.

Now, if $T z \neq z$, then as $g$ is strictly quasi-convex, we have $g(z) \leq g(\lambda T z+(1-\lambda) z)<$ $\max \{g(z), g(T z)\}=g(z)$, which is a contradiction.

Hence, $T z=z$.

Next, we consider the Banach space $X$ with the Opial property. 
Theorem 3.11 Let $C$ be a nonempty subset of a Banach space $X$ having the Opial property. Let $T$ be a self-mapping on $C$ satisfying the condition $B_{\gamma, \mu}$. If $\left\{x_{n}\right\}$ is a sequence in $X$ such that

(i) $\left\{x_{n}\right\}$ converges weakly to $z$,

(ii) $\lim _{n \rightarrow \infty}\left\|T x_{n}-x_{n}\right\|=0$,

then $T z=z$.

Proof By Proposition 3.5(ii), (for $\left.\gamma=\frac{c}{2}, c \in[0,1]\right)$

$$
\gamma\left\|x_{n}-T x_{n}\right\| \leq\left\|x_{n}-z\right\| \leq\left\|x_{n}-z\right\|+\mu\|z-T z\| .
$$

So, by the condition $B_{\gamma, \mu}$,

$$
\left\|T x_{n}-T z\right\| \leq(1-\gamma)\left\|x_{n}-z\right\|+\mu\left(\left\|x_{n}-T z\right\|+\left\|z-T x_{n}\right\|\right) .
$$

Now,

$$
\begin{aligned}
\left\|x_{n}-T z\right\| \leq & \left\|x_{n}-T x_{n}\right\|+\left\|T x_{n}-T z\right\| \\
\leq & \left\|x_{n}-T x_{n}\right\|+(1-\gamma)\left\|x_{n}-z\right\| \\
& +\mu\left(\left\|x_{n}-T z\right\|+\left\|z-x_{n}\right\|+\left\|x_{n}-T x_{n}\right\|\right) \quad(\text { by (3.3)). }
\end{aligned}
$$

So, taking limit as $n \longrightarrow \infty$ and using (ii), we get

$$
\begin{aligned}
\left\|x_{n}-T z\right\| & \leq \frac{1-\gamma+\mu}{1-\mu}\left\|x_{n}-z\right\| \\
& \leq\left\|x_{n}-z\right\| .
\end{aligned}
$$

So,

$$
\lim _{n \rightarrow \infty} \inf \left\|x_{n}-T z\right\| \leq \lim _{n \rightarrow \infty} \inf \left\|x_{n}-z\right\| .
$$

Let $T z \neq z$. Since $x_{n} \longrightarrow z$ (weakly), by the Opial property, we have

$$
\lim _{n \rightarrow \infty} \inf \left\|x_{n}-z\right\|<\lim _{n \rightarrow \infty} \inf \left\|x_{n}-T z\right\|,
$$

which is a contradiction to (3.4).

So, $T z=z$.

Example 3.12 We consider the set $C\left(\subset l^{p}, 1<p<\infty\right)$ where

$$
C=\left\{\left\{x_{n}\right\} \in l^{p}:\left|x_{1}\right| \leq \frac{1}{2}, x_{j}=0 \forall j \neq 1\right\} .
$$

Let $\left\{a_{n}\right\}$ be a sequence in $C$ such that

$$
a_{1}=\left\{\frac{1}{2}, 0,0,0 \ldots\right\}, \quad a_{2}=\left\{\frac{2}{3}, 0,0, \ldots\right\} \quad \ldots \quad a_{n+1}=\left\{\frac{n}{n+1}, 0,0 \ldots\right\} .
$$

Then $\left\{a_{n}\right\}$ converges to $z=\{1,0,0 \ldots\}$. 
Let $T: C \longrightarrow C$ be such that $T\left(\left\{a_{n}\right\}\right)=T\left(\left\{x_{1}, 0,0 \ldots\right\}\right)=\left\{x_{1}^{2}, 0,0 \ldots\right\}$.

Now, let $X_{1}=\left\{x_{1}, 0,0 \ldots\right\}, Y_{1}=\left\{y_{1}, 0,0 \ldots\right\} \in C$. Then

$$
\begin{aligned}
& \left\|T X_{1}-T Y_{1}\right\|_{p} \\
& \quad=\left\|\left\{x_{1}^{2}-y_{1}^{2}, 0,0, \ldots\right\}\right\|_{p} \\
& \quad=\left|x_{1}^{2}-y_{1}^{2}\right| \leq\left(\left|x_{1}\right|+\left|y_{1}\right|\right)\left|x_{1}-y_{1}\right| \\
& \quad \leq\left|x_{1}-y_{1}\right|=\left\|X_{1}-Y_{1}\right\|_{p} .
\end{aligned}
$$

So, $T$ is nonexpansive and hence satisfies the condition $B_{\gamma, \mu}$.

Again,

$$
\begin{aligned}
\lim _{n \rightarrow \infty}\left\|T X_{n}-X_{n}\right\|_{p} \\
\quad=\lim _{n \rightarrow \infty}\left\|\left\{\left(\frac{n}{n+1}\right)^{2}-\left(\frac{n}{n+1}\right), 0,0 \ldots \ldots\right\}\right\|_{p} \\
=\lim _{n \rightarrow \infty}\left|\left(\frac{n}{n+1}\right)^{2}-\left(\frac{n}{n+1}\right)\right| \\
=0 .
\end{aligned}
$$

Thus, all the conditions of Theorem 3.11 are satisfied. Hence, $T z=z$. (Clearly, $z=$ $\{1,0,0 \ldots\}$ is a fixed point of $T$.)

In the following result, we take $C$ as a weakly compact and convex subset.

Theorem 3.13 Let $C$ be a weakly compact convex subset of a Banach space $X$ with the Opial property, $T$ be a self-mapping on $C$ satisfying the condition $B_{\gamma, \mu}$, and the sequence $\left\{x_{n}\right\}$ in $C$ be as defined in Proposition 3.6. Then $\left\{x_{n}\right\}$ converges weakly to a fixed point of $T$.

Proof By Proposition 3.6, $\left\|T x_{n}-x_{n}\right\| \longrightarrow 0$ as $n \longrightarrow \infty$.

Since $C$ is weakly compact, there exists a subsequence $\left\{x_{n_{j}}\right\}$ of $\left\{x_{n}\right\}$ and $z \in C$ such that $\left\{x_{n_{j}}\right\}$ converges weakly to $z$. Now, by Theorem $3.11, z$ is a fixed point of $T$.

We assume that $\left\{x_{n}\right\}$ does not converge weakly to $z$. Then there is a subsequence $\left\{x_{n_{k}}\right\}$ of $\left\{x_{n}\right\}$ and $u \in C$ such that $\left\{x_{n_{k}}\right\}$ converges weakly to $u$ and $u \neq z$. Again, $T u=u$ (by Theorem 3.11).

Now,

$$
\begin{aligned}
\lim _{n \rightarrow \infty} \inf \left\|x_{n}-z\right\| \\
\quad=\lim _{n_{j} \rightarrow \infty} \inf \left\|x_{n_{j}}-z\right\| \\
\quad<\lim _{n_{j} \rightarrow \infty} \inf \left\|x_{n_{j}}-u\right\| \quad \text { (by Opial property) } \\
\quad=\lim _{n_{k} \rightarrow \infty} \inf \left\|x_{n_{k}}-u\right\| \\
\quad<\lim _{n_{k} \rightarrow \infty} \inf \left\|x_{n_{k}}-z\right\|
\end{aligned}
$$




$$
=\lim _{n \rightarrow \infty} \inf \left\|x_{n}-z\right\|
$$

which is a contradiction.

So, $\left\{x_{n}\right\}$ converges weakly to $z$.

Example 3.14 We consider the same set $C$ and the self-mapping $T$ as in Example 3.12. For $X_{0}=\left\{\frac{1}{2}, 0,0 \ldots\right\} \in C$, let the sequence $\left\{X_{n}\right\}$ in $C$ be defined by

$$
X_{n+1}=\lambda T X_{n}+(1-\lambda) X_{n}, \quad n \in \mathbb{N} \cup\{0\}, \lambda \in[\gamma, 1)-\{0\},
$$

which converges weakly to a fixed point of $T$ by Theorem 3.13 .

Now

$$
\begin{aligned}
& X_{1}=\left\{\frac{\lambda}{2^{2}}+(1-\lambda) \frac{1}{2}, 0,0, \ldots\right\}, \\
& X_{2}=\left\{\lambda\left(\frac{\lambda}{2^{2}}+(1-\lambda) \frac{1}{2}\right)^{2}+(1-\lambda)\left(\frac{\lambda}{2^{2}}+(1-\lambda) \frac{1}{2}\right), 0,0, \ldots\right\}, \quad \text { etc. }
\end{aligned}
$$

Clearly, $\left\{X_{n}\right\}$ converges to $\{0,0,0, \ldots\}$ which is a fixed point of $T$ in $C$.

Similar result as in Theorem 3.13 can be obtained by taking $C$ as a closed, convex, and bounded subset of a Banach space $X$ with the Opial property.

Next we discuss the convergence of some iteration schemes to the fixed point of $T$.

For a Banach space $X$, the Mann iteration scheme (see [29]) is defined by: $x_{0} \in X, x_{n+1}=$ $\left(1-\alpha_{n}\right) x_{n}+\alpha_{n} T x_{n}, n \in \mathbb{N} \cup\{0\}, 0 \leq \alpha_{n} \leq 1$, for each $n, \sum \alpha_{n}=\infty$.

Using Theorem 3.8, we can see the convergence of the above iteration scheme to the fixed point of $T$.

Here, we discuss the convergence of the following type of extended Mann iteration scheme:

$$
\begin{aligned}
& x_{0} \in X, \\
& y_{n}=\alpha_{n} T x_{n}+\left(1-\alpha_{n}\right) x_{n}, \\
& x_{n+1}=\beta_{n} T y_{n}+\left(1-\beta_{n}\right) y_{n}, \\
& n \in \mathbb{N} \cup\{0\}, 0 \leq \alpha_{n}, \beta_{n} \leq 1, \text { for each } n, \text { and } \sum \alpha_{n}=\infty, \sum \beta_{n}=\infty .
\end{aligned}
$$

Lemma 3.15 Let $C$ be a nonempty closed and convex subset of a Banach space $X$. Let $T$ be a self-mapping on $C$ satisfying the condition $B_{\gamma, \mu}$. For $x_{0} \in C$, let $\left\{x_{n}\right\}$ be the sequence in $C$ defined by the above iteration scheme (3.5). Then $\lim _{n \rightarrow \infty}\left\|x_{n}-z\right\|$ exists for all $z \in F(T)$.

Proof Let $F(T) \neq \phi$, and let $z \in F(T)$.

By Lemma 3.3, $\left\|z-T x_{n}\right\| \leq\left\|z-x_{n}\right\| \forall n \in \mathbb{N} \cup\{0\}$.

Now,

$$
\begin{aligned}
\left\|x_{n+1}-z\right\| & =\left\|\beta_{n} T y_{n}+\left(1-\beta_{n}\right) y_{n}-z\right\| \\
& \leq \beta_{n}\left\|T y_{n}-z\right\|+\left(1-\beta_{n}\right)\left\|y_{n}-z\right\| \\
& \leq \beta_{n}\left\|y_{n}-z\right\|+\left(1-\beta_{n}\right)\left\|y_{n}-z\right\|
\end{aligned}
$$




$$
\begin{aligned}
& =\left\|y_{n}-z\right\| \\
& =\left\|\alpha_{n} T x_{n}+\left(1-\alpha_{n}\right) x_{n}-z\right\| \\
& \leq \alpha_{n}\left\|x_{n}-z\right\|+\left(1-\alpha_{n}\right)\left\|x_{n}-z\right\| \\
& =\left\|x_{n}-z\right\|,
\end{aligned}
$$

i.e., $\left\{\left\|x_{n}-z\right\|\right\}$ is a nonincreasing and bounded sequence.

Thus $\lim _{n \rightarrow \infty}\left\|x_{n}-z\right\|$ exists for all $z \in F(T)$.

Lemma 3.16 ([2]) Let $X$ be a uniformly convex Banach space. Let $\left\{\lambda_{n}\right\}$ be a sequence of real numbers such that $0<a \leq \lambda_{n} \leq b<1 \forall n \in \mathbb{N}$, and let $\left\{x_{n}\right\}$ and $\left\{y_{n}\right\}$ be sequences in $X$ such that $\lim _{n \rightarrow \infty} \sup \left\|x_{n}\right\| \leq r, \lim _{n \rightarrow \infty} \sup \left\|y_{n}\right\| \leq r$, and $\lim _{n \rightarrow \infty}\left\|\lambda_{n} x_{n}+\left(1-\lambda_{n}\right) y_{n}\right\|=r$ for some $r \geq 0$. Then $\lim _{n \rightarrow \infty}\left\|x_{n}-y_{n}\right\|=0$.

Theorem 3.17 Let $C$ be a nonempty closed convex subset of a uniformly convex Banach space $X$. Let $T$ be a self-mapping on $C$ satisfying $B_{\gamma, \mu}$ condition. Let $\left\{x_{n}\right\}$ be a sequence in $C$ defined by the iteration scheme (3.5) where $\alpha_{n}, \beta_{n} \in(0,1)$. Then $F(T) \neq \phi$ if and only if $\left\{x_{n}\right\}$ is bounded and $\lim _{n \rightarrow \infty}\left\|T x_{n}-x_{n}\right\|=0$.

Proof Let $F(T) \neq \phi$ and $z \in F(T)$.

By Lemma 3.15, $\lim _{n \rightarrow \infty}\left\|x_{n}-z\right\|$ exists and $\left\{x_{n}\right\}$ is bounded.

$$
\lim _{n \rightarrow \infty}\left\|x_{n}-z\right\|=p \quad \text { (say) }
$$

Now,

$$
\begin{aligned}
\left\|T y_{n}-z\right\| & \leq\left\|y_{n}-z\right\| \quad \text { (by Lemma 3.3) } \\
& \leq\left\|x_{n}-z\right\| .
\end{aligned}
$$

So,

$$
\lim _{n \rightarrow \infty} \sup \left\|T y_{n}-z\right\| \leq \lim _{n \rightarrow \infty} \sup \left\|y_{n}-z\right\| \leq \lim _{n \rightarrow \infty} \sup \left\|x_{n}-z\right\|=p .
$$

Also,

$$
\begin{aligned}
& \lim _{n \rightarrow \infty}\left\|\beta_{n}\left(T y_{n}-z\right)+\left(1-\beta_{n}\right)\left(y_{n}-z\right)\right\| \\
& \quad=\lim _{n \rightarrow \infty}\left\|x_{n+1}-z\right\|=p .
\end{aligned}
$$

So, by Lemma 3.16, $\lim _{n \rightarrow \infty}\left\|T y_{n}-y_{n}\right\|=0$.

Again, by Lemma 3.3 we have

$$
\lim _{n \rightarrow \infty} \sup \left\|T x_{n}-z\right\| \leq \lim _{n \rightarrow \infty} \sup \left\|x_{n}-z\right\|=p .
$$

Now,

$$
\left\|x_{n+1}-z\right\| \leq \beta_{n}\left\|T y_{n}-z\right\|+\left(1-\beta_{n}\right)\left\|y_{n}-z\right\|
$$




$$
\begin{aligned}
& \leq \beta_{n}\left\|y_{n}-z\right\|+\left(1-\beta_{n}\right)\left\|y_{n}-z\right\| \\
& =\left\|y_{n}-z\right\| \forall n \in \mathbb{N} \cup\{0\} \\
\Rightarrow \quad p & \leq \lim _{n \rightarrow \infty} \inf \left\|y_{n}-z\right\| \\
& \leq \lim _{n \rightarrow \infty} \sup \left\|y_{n}-z\right\| \leq p \quad(\text { using (3.7)) } \\
\Rightarrow \quad & \lim _{n \rightarrow \infty}\left\|y_{n}-z\right\|=p .
\end{aligned}
$$

So,

$$
\lim _{n \rightarrow \infty}\left\|\alpha_{n}\left(T x_{n}-z\right)+\left(1-\alpha_{n}\right)\left(x_{n}-z\right)\right\|=\lim _{n \rightarrow \infty}\left\|y_{n}-z\right\|=p .
$$

Using (3.6), (3.8), and (3.9) with Lemma 3.16, we get

$$
\begin{gathered}
\lim _{n \rightarrow \infty}\left\|\left(T x_{n}-z\right)-\left(x_{n}-z\right)\right\|=0 \\
\Rightarrow \quad \lim _{n \rightarrow \infty}\left\|T x_{n}-x_{n}\right\|=0 .
\end{gathered}
$$

Conversely, let $\left\{x_{n}\right\}$ be bounded and $\lim _{n \rightarrow \infty}\left\|T x_{n}-x_{n}\right\|=0$. Let $z \in A\left(C,\left\{x_{n}\right\}\right)$. By Proposition 3.5(iii), for, $\gamma=\frac{c}{2}, c \in[0,1]$,

$$
\begin{aligned}
\left\|x_{n}-T z\right\| \leq & (3-c)\left\|x_{n}-T x_{n}\right\|+\left(1-\frac{c}{2}\right)\left\|x_{n}-z\right\|+\mu\left(2\left\|x_{n}-T x_{n}\right\|\right. \\
& \left.+\left\|x_{n}-T z\right\|+\left\|z-T x_{n}\right\|+2\left\|T x_{n}-T^{2} x_{n}\right\|\right) \\
\leq & (3-c)\left\|x_{n}-T x_{n}\right\|+\left(1-\frac{c}{2}\right)\left\|x_{n}-z\right\|+\mu\left(2\left\|x_{n}-T x_{n}\right\|\right. \\
& \left.+\left\|x_{n}-T z\right\|+\left\|x_{n}-z\right\|+\left\|x_{n}-T x_{n}\right\|+2\left\|x_{n}-T x_{n}\right\|\right) \\
& (\text { by Proposition } 3.5(\mathrm{i})) \\
\Rightarrow \quad(1-\mu) \lim _{n \rightarrow \infty} \sup \left\|x_{n}-T z\right\| \leq\left(1-\frac{c}{2}+\mu\right) \lim _{n \rightarrow \infty} \sup \left\|x_{n}-z\right\| & \quad \leq \lim _{n \rightarrow \infty} \sup \left\|x_{n}-z\right\| \\
\Rightarrow \quad \lim _{n \rightarrow \infty} \sup \left\|x_{n}-T z\right\| \leq & \frac{1-\frac{c}{2}+\mu}{1-\mu} \lim _{n \rightarrow \infty} \sup \left\|x_{n}-z\right\| \\
& \quad\left(\operatorname{as} \frac{1-\frac{c}{2}+\mu}{1-\mu} \leq 1, \text { for } 2 \mu \leq \gamma=\frac{c}{2}\right) \\
\Rightarrow \quad & r\left(T z,\left\{x_{n}\right\}\right) \leq r\left(z,\left\{x_{n}\right\}\right) .
\end{aligned}
$$

So, $T z \in A\left(C,\left\{x_{n}\right\}\right)$.

Since $X$ is uniformly convex, so $T z=z$, i.e., $z \in F(T)$.

So, $F(T) \neq \phi$. 
Example 3.18 Let $T:[0,5] \longrightarrow[0,5]$ be defined by

$$
T(x)= \begin{cases}0 & \text { if } x \neq 5 \\ 2 & \text { if } x=5\end{cases}
$$

Then $T$ satisfies the condition $B_{\gamma, \mu}$.

For $x_{0}=2$, we construct the sequence $\left\{x_{n}\right\}$ by iteration scheme (3.5), where $\alpha_{n}=\alpha \in(0,1)$ and $\beta_{n}=\beta \in(0,1), n \in \mathbb{N} \cup\{0\}$.

Then we get $x_{n}=2(1-\alpha)^{n}(1-\beta)^{n}, n \geq 1$. Clearly $\left\{x_{n}\right\}$ is bounded.

$$
\text { Now, } \begin{aligned}
\lim _{n \rightarrow \infty}\left\|T x_{n}-x_{n}\right\| & =\lim _{n \rightarrow \infty}\left\|T\left(2(1-\alpha)^{n}(1-\beta)^{n}\right)-2(1-\alpha)^{n}(1-\beta)^{n}\right\| \\
& =\lim _{n \rightarrow \infty}|2(1-\alpha)(1-\beta)| \\
& =0 .
\end{aligned}
$$

Thus, by Theorem 3.17, $F(T) \neq \phi$. Clearly, $0 \in F(T)$ in this case.

Next we consider the following type of extended Picard-Mann hybrid iteration scheme:

$$
\begin{aligned}
& x_{0} \in X, \\
& y_{n}=\left(1-b_{n}\right) x_{n}+b_{n} T x_{n}, \\
& z_{n}=\left(1-a_{n}\right) x_{n}+a_{n} T y_{n}, \\
& x_{n+1}=T z_{n}, \\
& 0 \leq a_{n}, b_{n} \leq 1 \text { for each } n, \quad \sum_{n} a_{n}=\infty, \quad \sum_{n} b_{n}=\infty .
\end{aligned}
$$

Lemma 3.19 Let $T$ be a self-mapping on a nonempty closed and convex subset $C$ of a Banach space X. Let $T$ satisfy the condition $B_{\gamma, \mu}$ on $C$. For $x_{0} \in C$, we define a sequence $\left\{x_{n}\right\}$ in $C$ by the iteration scheme (3.10), where $0<a_{n}, b_{n}<1$. Then $\lim _{n \rightarrow \infty}\left\|x_{n}-z\right\|$ exists for all $z \in F(T)$.

Proof Similar as in Lemma 3.15.

Theorem 3.20 Let $T$ be a self-mapping on a nonempty closed and convex subset $C$ of a uniformly convex Banach space X. Let T satisfy the condition $B_{\gamma, \mu}$ on C. Let $\left\{x_{n}\right\}$ be a sequence in $C$ defined by the iteration scheme (3.10), where $0<a_{n}, b_{n}<1$ and $\lim _{n \rightarrow \infty} a_{n}=$ $k(\neq 0)$. Then $F(T) \neq \phi$ if and only if $\left\{x_{n}\right\}$ is bounded and $\lim _{n \rightarrow \infty}\left\|T x_{n}-x_{n}\right\|=0$.

Proof Let $F(T) \neq \phi$ and $z \in F(T)$. Then, by Lemma 3.19, $\lim _{n \rightarrow \infty}\left\|x_{n}-z\right\|$ exists and $\left\{x_{n}\right\}$ is bounded.

$$
\lim _{n \rightarrow \infty}\left\|x_{n}-z\right\|=p \quad \text { (say) }
$$

As in Theorem 3.17, we have

$$
\left\|T y_{n}-z\right\| \leq\left\|y_{n}-z\right\| \leq\left\|x_{n}-z\right\| .
$$


So,

$$
\lim _{n \rightarrow \infty} \sup \left\|T y_{n}-z\right\| \leq \lim _{n \rightarrow \infty} \sup \left\|y_{n}-z\right\| \leq \lim _{n \rightarrow \infty} \sup \left\|x_{n}-z\right\|=p .
$$

Again,

$$
\begin{aligned}
& \left\|x_{n+1}-z\right\| \\
& \quad=\left\|T z_{n}-z\right\| \leq\left\|z_{n}-z\right\| \\
& \quad=\left\|\left(1-a_{n}\right) x_{n}+a_{n} T y_{n}-z\right\| \\
& \quad \leq a_{n}\left\|T y_{n}-z\right\|+\left(1-a_{n}\right)\left\|x_{n}-z\right\| \\
& \quad \leq a_{n}\left\|y_{n}-z\right\|+\left(1-a_{n}\right)\left\|x_{n}-z\right\| \\
& \quad=a_{n}\left\|\left(1-b_{n}\right) x_{n}+b_{n} T x_{n}-z\right\|+\left(1-a_{n}\right)\left\|x_{n}-z\right\| \\
& \quad \leq a_{n}\left(b_{n}\left\|T x_{n}-z\right\|+\left(1-b_{n}\right)\left\|x_{n}-z\right\|\right)+\left(1-a_{n}\right)\left\|x_{n}-z\right\| \\
& \quad \leq a_{n} b_{n}\left\|x_{n}-z\right\|+a_{n}\left\|x_{n}-z\right\|-a_{n} b_{n}\left\|x_{n}-z\right\|+\left\|x_{n}-z\right\|-a_{n}\left\|x_{n}-z\right\| \\
& =\left\|x_{n}-z\right\| .
\end{aligned}
$$

Thus,

$$
\left\|x_{n+1}-z\right\| \leq\left\|x_{n}-z\right\|
$$

Again, by Lemma 3.3, we have

$$
\begin{aligned}
& \left\|T x_{n}-z\right\| \leq\left\|x_{n}-z\right\| \quad \text { for all } n \in \mathbb{N} \cup\{0\} \\
& \quad \Rightarrow \quad \lim _{n \rightarrow \infty} \sup \left\|T x_{n}-z\right\| \leq \lim _{n \rightarrow \infty} \sup \left\|x_{n}-z\right\|=p .
\end{aligned}
$$

Now, from equation (3.13), we have

$$
\begin{aligned}
& \left\|x_{n+1}-z\right\| \leq a_{n}\left\|y_{n}-z\right\|+\left(1-a_{n}\right)\left\|x_{n}-z\right\| \\
& \quad \Rightarrow \quad\left\|x_{n+1}-z\right\|-\left(1-a_{n}\right)\left\|x_{n}-z\right\| \leq a_{n}\left\|y_{n}-z\right\| \\
& \Rightarrow \quad p-(1-k) p \leq k \lim _{n \rightarrow \infty} \inf \left\|y_{n}-z\right\| \\
& \Rightarrow \quad p \leq \lim _{n \rightarrow \infty} \inf \left\|y_{n}-z\right\| \lim _{n \rightarrow \infty} \sup \left\|y_{n}-z\right\| \leq p \quad \text { (by (3.12)) } \\
& \Rightarrow \quad \lim _{n \rightarrow \infty}\left\|y_{n}-z\right\|=p .
\end{aligned}
$$

Thus,

$$
\lim _{n \rightarrow \infty}\left\|b_{n}\left(T x_{n}-z\right)+\left(1-b_{n}\right)\left(x_{n}-z\right)\right\|=\lim _{n \rightarrow \infty}\left\|y_{n}-z\right\|=p .
$$

By (3.11), (3.15), (3.16) and Lemma 3.16, we have $\lim _{n \rightarrow \infty}\left\|T x_{n}-x_{n}\right\|=0$.

For the converse part, let $\left\{x_{n}\right\}$ be bounded and $\lim _{n \rightarrow \infty}\left\|T x_{n}-x_{n}\right\|=0$. 
Let $z \in A\left(C,\left\{x_{n}\right\}\right)$. Then, using Proposition 3.5(iii) and following Theorem 3.17, for $\gamma=$ $\frac{c}{2}, c \in[0,1]$, we have

$$
\begin{aligned}
\lim _{n \rightarrow \infty} \sup \left\|x_{n}-T z\right\| & \leq \frac{1-\frac{c}{2}+\mu}{1-\mu} \lim _{n \rightarrow \infty} \sup \left\|x_{n}-z\right\| \\
& \leq \lim _{n \rightarrow \infty} \sup \left\|x_{n}-z\right\| \quad\left(\text { as } \frac{1-\frac{c}{2}+\mu}{1-\mu} \leq 1, \text { for } 2 \mu \leq \gamma=\frac{c}{2}\right) \\
\Rightarrow r\left(T z,\left\{x_{n}\right\}\right) & \leq r\left(z,\left\{x_{n}\right\}\right) .
\end{aligned}
$$

Hence, $T z \in A\left(C,\left\{x_{n}\right\}\right)$.

$X$ being uniformly convex, $T z=z$, i.e., $z \in F(T)$.

Hence, $F(T) \neq \phi$.

Example 3.21 We consider the same set $C$ with the self-mapping $T$ as in Example 3.12.

For $X_{0}=\left\{\frac{1}{3}, 0,0,0 \ldots\right\} \in C$, we construct the sequence $\left\{X_{n}\right\}$ in $C$ by the iteration scheme (3.10).

Let $a_{n}=a$ and $b_{n}=b \forall n \in \mathbb{N} \cup\{0\}$. Then

$$
\begin{aligned}
& Y_{0}=(1-b)\left\{\frac{1}{3}, 0,0,0 \ldots\right\}+b\left\{\frac{1}{3^{2}}, 0,0,0 \ldots\right\}, \\
& Z_{0}=(1-a)\left\{\frac{1}{3}, 0,0,0 \ldots\right\}+a\left\{\left((1-b) \frac{1}{3}+b \frac{1}{3^{2}}\right)^{2}, 0,0,0 \ldots\right\}, \\
& X_{1}=\left\{\left((1-a) \frac{1}{3}+a\left((1-b) \frac{1}{3}+b \frac{1}{3^{2}}\right)^{2}\right)^{2}, 0,0, \ldots\right\}, \quad \text { and so on. }
\end{aligned}
$$

Thus, it can be seen that $\left\{X_{n}\right\}$ is bounded and $\lim _{n \rightarrow \infty}\left\|T X_{n}-X_{n}\right\|=0$. Hence, by Theorem 3.20, $F(T) \neq \phi$ (here, clearly, $\{0,0, \ldots\} \in F(T)$ ).

\section{Conclusion}

Throughout the paper, we have discussed some fixed point results for the class of mappings with $B_{\gamma, \mu}$ condition. In 2010, Harandi and Emami (see [7]) studied some fixed point theorems for contraction type mappings in partially ordered metric spaces with applications in solving ordinary differential equations. In this context, the study of fixed point theory in partially ordered metric spaces for the class of mappings with $B_{\gamma, \mu}$ condition with different practical applications is a scope for future study.

\section{Acknowledgements}

The authors thank anonymous referees for their careful reading and suggestions.

Funding

Not applicable.

\section{Abbreviations}

Not applicable.

Availability of data and materials

Not applicable. 
Authors' contributions

All authors contributed equally to the writing of this paper. All authors read and approved the final manuscript.

\section{Author details}

${ }^{1}$ Department of Mathematics, Gauhati University, Guwahati, India. ${ }^{2}$ Department of Mathematics, Indira Gandhi National Tribal University, Amarkantak, India.

\section{Publisher's Note}

Springer Nature remains neutral with regard to jurisdictional claims in published maps and institutional affiliations.

\section{Received: 27 March 2018 Accepted: 27 July 2018 Published online: 13 August 2018}

\section{References}

1. Afshari, H., Aydi, H., Karapınar, E.: Existence of fixed points of set-valued mappings in b-metric spaces. East Asian Math. J. 32, 319-332 (2016)

2. Akkasriworm, N.: Fixed point theorems and approximating fixed points of generalised (C)-condition mappings. Int. J. Math. Anal. 7(19), 915-925 (2013)

3. Aksoy, U., Karapınar, E., Erhan, I.M.: Fixed points of generalized alpha-admissible contractions on b-metric spaces with an application to boundary value problems. J. Nonlinear Convex Anal. 17, 1095-1108 (2016)

4. Aksoy, U., Karapinar, E., Erhan, I.M.: Fixed point theorems in complete modular metric spaces and an application to anti-periodic boundary value problems. Filomat 31(17), 5475-5488 (2017)

5. Ali, M.U., Kamran, T., Karapinar, E.: Fixed point of $\alpha-\phi$-contractive type mappings in uniform spaces. Fixed Point Theory Appl. 2014, 150 (2014)

6. Alqahtani, B., Fulga, A., Karapınar, E.: Non-unique fixed point results in extended b-metric space. Mathematics 6(5), 68 (2018) https://doi.org/10.3390/math6050068

7. Amini-Harandi, A., Emami, H.: A fixed point theorem for contraction type maps in partially ordered metric spaces and application to ordinary differential equations. Nonlinear Anal. 72, 2238-2242 (2010)

8. Benavides, T.D., Medina Perez, B.: The fixed point property for some generalized non-expansive mappings senormings. J. Math. Anal. Appl. 429, 800-813 (2015)

9. Das, D., Goswami, N.: Some fixed point theorems on the sum and product of operators in tensor product spaces. Int. J. Pure Appl. Math. 109(3), 651-663 (2016)

10. Das, D., Goswami, N., Mishra, V.N.: Some results on fixed point theorems in Banach algebras. Int. J. Anal. Appl. 13(1), $32-40$ (2017)

11. Das, D., Goswami, N., Mishra, V.N.: Some results on the projective cone normed tensor product spaces over Banach algebras. Bol. Soc. Parana. Mat. 38(1), 197-221 (2020) (to appear)

12. Deepmala: A study on fixed point theorems for nonlinear contractions and its applications. Ph.D. thesis, Pt. Ravishankar Shukla University (2014)

13. Deepmala, Agarwal, R.P.: Existence and uniqueness of solutions for certain functional equations and system of functional equations arising in dynamic programming. An. Ştiinţ. Univ. 'Ovidius' Constanţa, Ser. Mat. 24(1), 3-28 (2016)

14. Dhompongsa, S., Inthakon, W., Kaewkhao, A.: Edelstein's method and fixed point theorems for some generalised non-expansive mappings. J. Math. Anal. Appl. 350, 12-17 (2009)

15. Falset, J.G., Fuster, E.L., Suzuki, T.: Fixed point theory for a class of generalised non-expansive mappings. J. Math. Anal. Appl. 375, 185-195 (2011)

16. Kanas, S., Srivastava, H.M.: Linear operators associated with k-uniformly convex functions. Integral Transforms Spec. Funct. 9(2), 121-132 (2000)

17. Karapinar, E.: Remarks on Suzuki (C)-condition. Atilim University, Department of Mathematics, Incek, 06836 Ankara, Turkey

18. Karapinar, E., Tas, K.: Generalised (C)-conditions and related fixed point theorems. Comput. Math. Appl. 61, 3370-3380 (2011)

19. Khamsi, M.A., Khan, A.R.: On monotone nonexpansive mappings in $L_{1}([0,1])$. Fixed Point Theory Appl. 2015, Article ID 94 (2015). https://doi.org/10.1186/s13663-015-0346-x

20. Lael, F., Heidarpour, Z:: Fixed point theorems for a class of generalized non-expansive mappings. Fixed Point Theory Appl. 2016, 82 (2016)

21. Mishra, L.N.: On existence and behavior of solutions to some nonlinear integral equations with applications. Ph.D. thesis, National Institute of Technology (2017)

22. Mishra, L.N., Jyoti, K., Rani, A., Vandana: Fixed point theorems with digital contractions image processing. Nonlinear Sci. Lett. A, Math. Phys. Mech. 9(2), 104-115 (2018)

23. Mishra, L.N., Tiwari, S.K., Mishra, V.N.: Fixed point theorems for generalized weakly S-contractive mappings in partial metric spaces. J. Appl. Anal. Comput. 5(4), 600-612 (2015). https://doi.org/10.11948/2015047

24. Mishra, L.N., Tiwari, S.K., Mishra, V.N., Khan, I.A.: Unique fixed point theorems for generalized contractive mappings in partial metric spaces. J. Funct. Spaces 2015, Article ID 960827 (2015)

25. Mishra, V.N.: Some problems on approximations of functions in Banach spaces. Ph.D. Thesis, Indian Institute of Technology (2007)

26. Pathak, H.K., Deepmala: Common fixed point theorems for PD-operator pairs under relaxed conditions with applications. J. Comput. Appl. Math. 239, 103-113 (2013)

27. Patir, B., Goswami, N., Mishra, L.N.: Fixed point theorems in fuzzy metric spaces for mappings with some contractive type conditions. Korean J. Math. 26(2), 307-326 (2018). https://doi.org/10.11568/kjm.2018.26.2.307

28. Srivastava, H.M., Mishra, A.K.: Applications of fractional calculus to parabolic starlike and uniformly convex functions Comput. Math. Appl. 39(3-4), 57-69 (2000) 
29. Sukla, R., Pant, R., De la Sen, M.: Generalized $\alpha$-non-expansive mappings in Banach spaces. Fixed Point Theory Appl. 2017, 4 (2017)

30. Suzuki, T: Strong convergence theorems for infinite families of non expansive mappings in general Banach spaces. Fixed Point Theory Appl. 2005, 685918 (2005)

31. Suzuki, T.: Fixed point theorems and convergence theorems for some generalized non- expansive mappings. J. Math. Anal. Appl. 340, 1088-1095 (2008)

32. Thakur, D., Thakur, B.S., Postolache, M.: Convergence theorems for generalized nonexpansive mappings in uniformly convex Banach spaces. Fixed Point Theory Appl. 2015, 144 (2015)

Submit your manuscript to a SpringerOpen ${ }^{\circ}$ journal and benefit from:

- Convenient online submission

- Rigorous peer review

- Open access: articles freely available online

- High visibility within the field

- Retaining the copyright to your article

Submit your next manuscript at $\boldsymbol{~ s p r i n g e r o p e n . c o m ~}$ 